\title{
TRES NUEVOS REGISTROS DE AGARICOMYCETES PARA LA ARGENTINA: CORTINARIUS CASIMIRI VAR. HOFFMANNII, LACTARIUS HEPATICUS Y MYCENA MARGARITA
}

\begin{abstract}
Agustín P. Martínez ${ }^{1}$, Juan M. Suarez ${ }^{1}$ \& Bernardo E. Lechner ${ }^{1,2}$
${ }^{1}$ Departamento de Biodiversidad y Biología Experimental, Facultad de Ciencias Exactas y Naturales, Universidad de Buenos Aires, Pabellón 2, Laboratorio 7, Intendente Güiraldes 2160, 1428 Ciudad Autónoma de Buenos Aires, Argentina. ${ }^{2}$ Instituto de Micología y Botánica (UBA-CONICET), Facultad de Ciencias Exactas y Naturales, Universidad de Buenos Aires, Pabellón 2, Laboratorio 7, Intendente Güiraldes 2160, 1428 Ciudad Autónoma de Buenos Aires Argentina; blechner@bg.fcen.uba.ar (autor corresponsal).
\end{abstract}

Abstract. Martínez, A. P.; J. M. Suarez \& B. E. Lechner. Three new records of Agaricomycetes for Argentina: Cortinarius casimiri var. hoffmannii, Lactarius hepaticus and Mycena margarita. Darwiniana, nueva serie 8(2): 438-448.

Three new records of Agaricomycetes are cited for the first time for Argentina: Cortinarius casimiri var. hoffmannii, Lactarius hepaticus and Mycena margarita. Complete descriptions and illustrations are provided in this work, and color photographs of the fresh samples were added. Comments were made about the taxonomy and ecology of the species presented.

Keywords. Agaricales; Cortinariaceae; Diversity; Mycenaceae; Russulaceae.

Resumen. Martínez, A. P.; J. M. Suarez \& B. E. Lechner. Tres nuevos registros de Agaricomycetes para la Argentina: Cortinarius casimiri var. hoffmannii, Lactarius hepaticus y Mycena margarita. Darwiniana, nueva serie 8(2): 438-448.

Se citan por primera vez tres nuevas especies de Agaricomycetes para la Argentina: Cortinarius casimiri var. hoffmannii, Lactarius hepaticus y Mycena margarita. En el presente trabajo se proveen descripciones completas e ilustraciones de las mismas con fotografías en color de las muestras frescas. Se realizan comentarios acerca de la taxonomía y ecología de las especies presentadas.

Palabras clave. Agaricales; Cortinariaceae; Diversidad; Mycenaceae; Russulaceae.

\section{INTRODUCCIÓN}

El estudio de la biodiversidad de los hongos Agaricales en la Argentina dista de ser completo. Muchos géneros se encuentran poco explorados y con muchas especies de aparición frecuente. Los Agaricales de la Argentina son uno de los grupos más estudiados en Sudamérica. Spegazzini y Singer, dos relevantes micólogos, coleccionaron y estudiaron los hongos de la Argentina y sus publicaciones enriquecieron el conocimiento del grupo en la región (Mueller \& $\mathrm{Wu}, 1997)$. Trabajos posteriores han descripto e ilustrado especies de Agaricales de la Argentina (p.e. Albertó, 1998; Lechner et al., 2003; 2004; 2005; 2006; Lechner \& Albertó, 2008).

El género Cortinarius (Pers.) Grey (Cortinariaceae, Agaricales) es uno de los más numerosos dentro del orden de los Agaricales. En la Argentina fue estudiado por Spegazzini (1887; 1888 y 1899), Singer \& Digilio (1952), Singer (1954; 1969), Moser \& Horak (1975), Horak (1980), Moser (2001) y Salgado Salomón et al. (2018), encontrando así cerca de 230 especies, que en su gran mayoría pertenecen a colecciones de la Patagonia (Romano \& Lechner, 2013; Niveiro \& Albertó, 2014). El género Lactarius Pers. (Russulaceae, Russulales), es un género con pocas 
citas para la Argentina en relación a lo que se conoce mundialmente, ya que sólo se han encontrado seis especies distintas en todo el país (Niveiro \& Albertó, 2013). El género Mycena (Pers.) Roussel (Mycenaceae, Agaricales), posee cerca de 100 especies en la Argentina. Fue estudiado, entre otros, por Singer, Raithlelhuber y Spegazzini (Niveiro \& Albertó, 2012).

Alo largo de varios estudios sobre la biodiversidad de Agaricomycetes en el Partido de la Costa, y en la zona Oeste de Ciudad de Buenos Aires fueron halladas por primera vez en la Argentina tres nuevas especies: Cortinarius casimiri var. hoffmannii (Reumaux) Suár.-Sant. \& A. Ortega, Lactarius hepaticus Plowr. y Mycena margarita (Murrill) Murrill. El objetivo de este trabajo es presentar tres nuevos registros de hongos agaricoides para la Argentina, los cuales son descritos e ilustrados con dibujos y fotografías a color.

\section{MATERIALES Y MÉTODOS}

Las muestras frescas fueron coleccionadas en los partidos de la Costa, de Lomas de Zamora y de Moreno en la provincia de Buenos Aires, Argentina (Fig. 1), y fueron documentadas y preservadas según la metodología estándar empleada para Agaricales (Largent, 1986). Se realizaron fotografias en campo y anotaciones de sus características macroscópicas como forma, tamaño y color del píleo, laminillas y estípite. También se observaron reacciones sobre el basidioma fresco con $\mathrm{KOH} \mathrm{5 \%}$. Los colores se anotaron de acuerdo con Kornerup \& Wanscher (1987).

Paraladescripciónmicroscópicaseutilizóelmétodo de examinación estándar para Agaricales (Wright \& Albertó, 2002). Se realizaron cortes en: la superficie del píleo para observar la pileipellis; las laminillas para describir basidios, cistidios y esporas; y el estípite para caracterizar caulocistidios y estipitipellis.

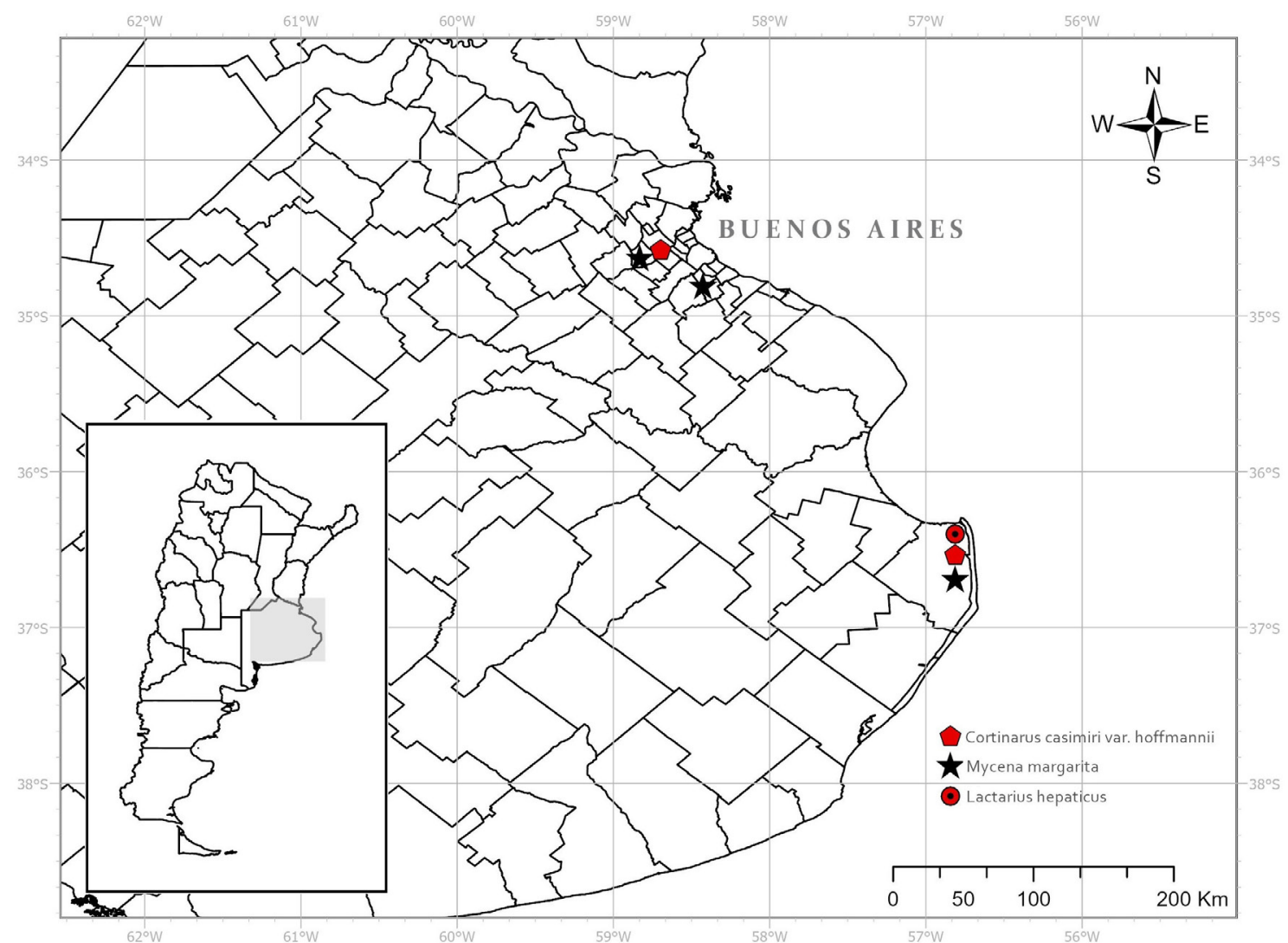

Fig. 1. Sitios de colección de los tres nuevos registros de Agaricomycetes en la provincia de Buenos Aires, Argentina. Figura en color en la versión en línea http:/www.ojs.darwin.edu.ar/index.php/darwiniana/article/view/888/1191 
Se utilizaron diferentes medios y tinciones para ayudar a la observación de estructuras y ornamentaciones: $\mathrm{KOH} 5 \%$, Floxina acuosa al 1\% y reactivo de Melzer.

Se utilizó el programa Piximètre (versión 5.10; www.piximetre.fr) para realizar la medición de todas las estructuras microscópicas, y se utilizaron los siguientes símbolos: $\mathrm{Q}=$ coeficiente entre el largo y el ancho de las esporas; $\mathrm{Qe}=$ media de los valores de $\mathrm{Q} ; \mathrm{N}=$ número de esporas medidas; $\mathrm{Me}$ $=$ tamaño promedio de las esporas.

Las colecciones fueron depositadas en el herbario micológico del Departamento de Biodiversidad y Biología Experimental de la Facultad de Ciencias Exactas y Naturales de la UBA (BAFC).

\section{TRATAMIENTO TAXONOMICO}

\section{Cortinarius casimiri var. hoffmannii (Reumaux)}

Suár--Sant. V. \& A. Ortega, Mycol. Res. 113 (10): 1080 (2009). Cortinarius decipiens var. hoffmannii Reumaux, Bull. trimest. Féd. Mycol. Dauphiné-Savoie 28 (no. 111): 24 (1988). TIPO: FRANCIA: Ardenas francesas: Bosque de Belval, "sous feuilles humides ous feuilles humides (cheênes, charmes, bouleaux)", 20 Sept. 1986, P. Reumaux (P.M.L. 559, como C. decipiens var. hoffmannii, holotipo, no visto). Figs. 2-3.

Píleo (Fig. 2) 15-45 mm diám., cónico a cónicocampanulado, luego convexo o plano-convexo, a veces con un umbón bien marcado, higrófano, castaño rojizo (11D4) a gris violáceo (12E3), blanquecino hacia el borde, aspecto lanoso debido a fibrillas blanquecinas dispuestas radialmente, más abundantes hacia el borde. Contexto delgado, menor a $4 \mathrm{~mm}$, ocráceo (9D6) hacia la superficie del píleo, pardo oscuro con tonalidades violáceas (13E4) cerca de las laminillas. Laminillas adnatas a sinuadas, subdistantes, con lamelas de distintos tamaños, ocre (7D5) con tonalidades gris rosáceas (12C3) o pardo violáceas (12C4) y finalmente de color castaño oscuro-ferroso (8C6). Estípite 20-40 x 3-5 mm, cilíndrico, a veces algo ensanchado en la base, gris-pardusco (16C1) con el tacto, cubierto de fibrillas blanquecinas longitudinales que no dejan diferenciar un anillo sino una banda dispersa hacia la mitad inferior, restos de cortina color ferroso (6E8), tonos gris rosáceos (12C3) hacia el ápice en ejemplares jóvenes, carne castaño grisácea (6D4). Esporada castaño clara (6E5). Olor no distintivo. Sabor no distinguible. Comestibilidad desconocida. Esporas (Fig. 3A) 7,3-9,3 (10,6) x 4,2-5,0 (5,6) $\mu \mathrm{m}$; $\mathrm{Q}=1,65-2 ; \mathrm{N}=50 ; \mathrm{Me}=8,4 \times 4,6 \mu \mathrm{m} ; \mathrm{Qe}=1,8$; castaño claras, elipsoidales, oblongo-elipsoidales, apiculadas, pared gruesa, verrugas irregulares, no amiloides. Basidios (Fig. 3B) 25,5-35,0 x 5,0$8,0 \mu \mathrm{m}$, claviformes, 4-esporados, fíbulados, con esterigmas de 2-4 $\mu \mathrm{m}$. Cistidios no se observaron. Trama himenoforal subparalela, hifas hialinas, pared delgada. Pileipellis (Fig. 3C) epicutis formado por hifas paralelas de 3-10 $\mu \mathrm{m}$ de diám., hipocutis bien diferenciado formado por hifas gruesas y cortas, cilíndricas o globosas, 14-30 $\mu \mathrm{m}$ de diám., con pigmento parietal ocre o pardo-ocre. Estipitipellis un epicutis formado por hifas paralelas de 3-6 $\mu \mathrm{m}$ de diám., no gelatinosas, lisas, cilíndricas, con pigmento parietal ocre, hipocutis formado por hifas paralelas de mayor tamaño, de 8-12 $\mu \mathrm{m}$ de diám., lisas, cilíndricas. Fíbulas presentes en todos los tejidos.

Hábito. Solitario o gregario, creciendo entre la hojarasca de caducifolios, generalmente cerca de Populus spp. Coleccionado generalmente en épocas de mucha lluvia y humedad.

Distribución y hábitat. Europa, principalmente en la región Eurosiberiana; generalmente bajo caducifolios y coníferas (Moënne-Loccoz \& Reumaux, 1990; Suárez-Santiago et al., 2009). Por tratarse de un género micorrícico asociado a planifolias Euroasiáticas, su hallazgo en la Argentina puede deberse a una introducción junto a Populus spp.

Observaciones. C. casimiri var. hoffmannii se caracteriza por poseer pequeños basidiomas higrófanos, con tonalidades rosadas en el pie, poseer una cutícula fibrillosa, generando una tonalidad pálida hacia el margen del píleo y un estípite fibrilloso que forma generalmente un pseudo anillo de velo remanente (Suárez-Santiago et al., 2009).

Esta variedad se diferencia de C. casimiri var. casimiri principalmente por su menor tamaño de esporas, 7,3-9,3 $\times$ 4,2-5,0 $\mu \mathrm{m}$ vs $10,3-12,0$ $\times 5,9-7,0 \mu \mathrm{m}$, respectivamente. Las diferencias macromorfológicas entre las distintas variedades son muy sutiles y estas características varían significativamente entre las distintas colecciones. 
A. P. MARTíneZ ET AL. Nuevos registros de Agaricomycetes para la Argentina

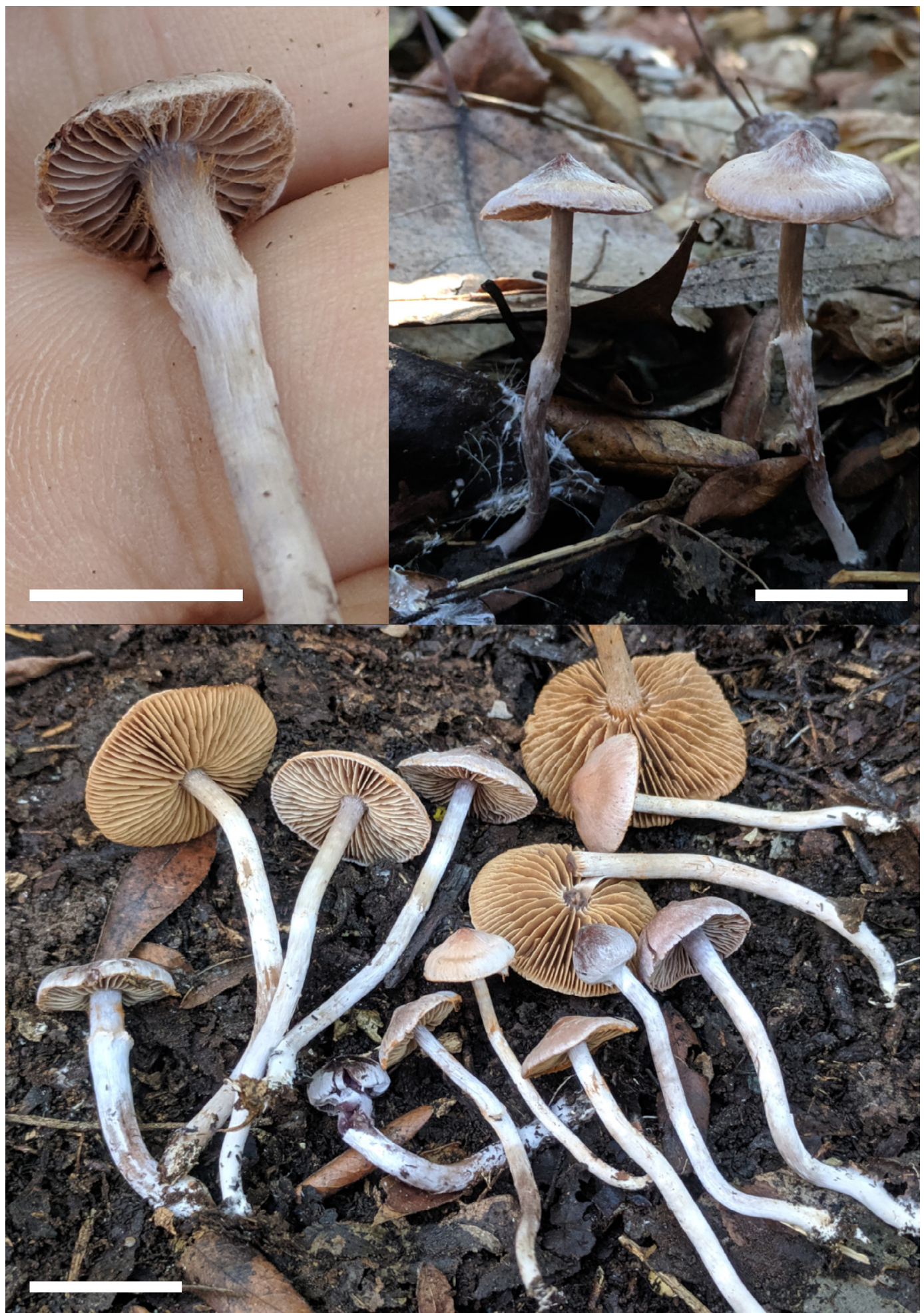

Fig. 2. Cortinarius casimiri var. hoffmani. Aspecto general. Escala: $20 \mathrm{~mm}$. Fotos Agustín P. Martínez. Figura en color en la versión en línea http://www.ojs.darwin.edu.ar/index.php/darwiniana/article/view/888/1191 

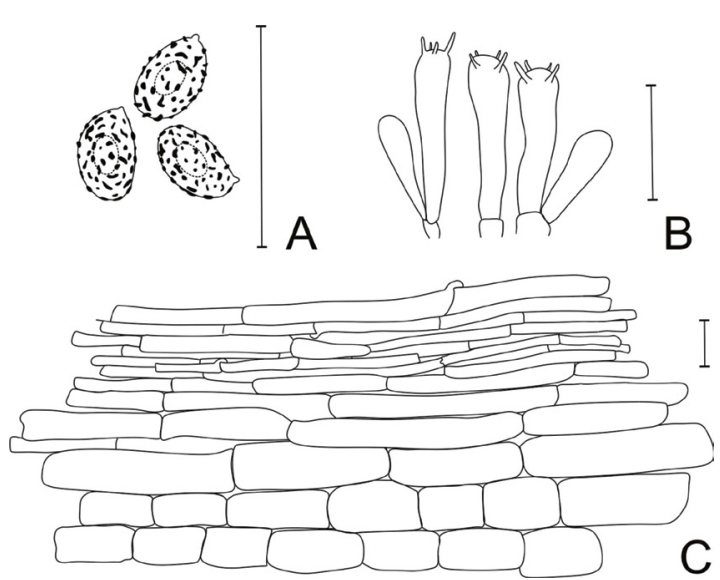

Fig. 3. Cortinarius casimiri var. hoffmani. A, esporas. B, basidios. C, pileipellis. Escala: $20 \mathrm{~mm}$. Ilustraciones Juan M. Suarez.

Antiguamente se consideraba a $C$. casimiri var. hoffmannii como una variedad de $C$. decipiens debido a su parecido macromorfológico y su similitud en el tamaño de esporas; sin embargo, se observó mediante análisis moleculares de ITS que la variedad hoffmannii se encuentra más relacionada con $C$. casimiri que con $C$. decipiens (SuárezSantiago et al., 2009), coincidiendo con la relación entre $C$. decipiens var. hoffmannii y $C$. casimiri planteada anteriormente por Arnold (1993).

Microscópicamente es difícil diferenciar a C. casimiri var. hoffmannii de C. decipiens, principalmente debido a que estos taxones presentan una alta variabilidad intra-específica de sus caracteres microscópicos (Niskanen et al., 2009); aunque, a partir de la observación del basidioma en fresco, se puede diferenciar $C$. casimiri var. hoffmannii debido a la presencia de tintes rojizos en el píleo, la ausencia de tintes violáceos en las laminillas, más notable en ejemplares jóvenes, y por el engrosamiento en la base del estípite, no necesariamente presente en todos los basidiomas.

El género Cortinarius se encuentra en la sección Hydrocybe, de la cual se conoce una distribución pequeña y acotada a regiones de Europa. En la Provincia de Buenos Aires C. casimiri var. hoffmannii es el segundo registro de este género, luego de Cortinarius varius. C. casimiri var. hoffmannii es un taxón poco estudiado y con pocos registros en el mundo.

\section{Material estudiado}

ARGENTINA, Buenos Aires, Moreno, Reserva Los Robles, $34^{\circ} 40^{\prime} 2^{\prime \prime} \mathrm{S} 58^{\circ}$ 51' 38" O, 28-mayo19, Leg. A. P. Martínez, BAFC 53411; Reserva Los Robles, 34 40' 7” S 58 51'39” O, 14-mayo-2019, Leg. A. P. Martínez, BAFC 53412; Ib. 34 40' 10” S 58 51' 42" O, 28-mayo-2019, Leg. A. P. Martínez, BAFC 53413; Partido de la Costa, San Bernardo, 36 36' 57" S 56 41' 11" O, 15-jun.-19, Leg J. M. Suarez \& A. P. Martínez, BAFC 53414.

Lactarius hepaticus Plowr., Icon. Mycol. 4: 28 (1: pl. 56). 1905. TIPO: Lámina 56, Icon. Mycol. (Paris) Tomo I. Figs. 4-5.

Píleo (Fig. 4) 18-40 mm, inicialmente convexo, luego plano, generalmente deprimido en la parte central, umbón pequeño y ligeramente deprimido en el centro; margen delgado, entero, ligeramente involuto cuando joven, coloración rojizo pálida (7A4); cutícula no separable, ligeramente arrugado en el área central, sepia castaño oscuro (9D5), castaño rojizo oscuro (9E7), castaño púrpura (10E8) a veces con reflejos oliva. Contexto escaso, menor a $5 \mathrm{~mm}$, crémeo (5A3), pálido, amarilleando ligeramente al corte. Laminillas adnatas a sub-decurrentes, apretadas, numerosas lamélulas de diferente longitud, anastomosadas, rosa crémeas (6A3) a castaño ocre (7D8). Estípite 43-80 x 6-10 mm, cilíndrico, frecuentemente curvado, especialmente en el tercio inferior, fistuloso; superficie finamente arrugada longitudinalmente hasta el tercio superior, luego lisa, de color crema parduzco (8B3) con tonalidades rosadas (8C5) a castaño rojizas (8D5). Látex no abundante, blanco (4A1), cambiando rápidamente a amarillo (4A4) cuando se aísla en vidrio y lentamente en contacto con carne o láminas; sabor acre. Esporada crémea (8A2). Olor no distintivo. Sabor suave, a veces amargo y/o picante. Comestibilidad posible toxicidad, sin valor culinario. Reacción química con hidróxido de potasio $5 \%(\mathrm{KOH})$ la carne se torna color pardo olivácea (4D3). Esporas (Fig. 5A) 8,9-10,5 × 7,0-8,2 (9,7) $\mu \mathrm{m} ; \mathrm{Me}=9,7 \times 7,6 \mu \mathrm{m} ; \mathrm{N}=30 ; \mathrm{Q}=1,1-1,4$, $\mathrm{Qe}=1,3$, hialinas, subglobosas a elipsoidales, verrugosas, reticuladas de manera casi completa, amiloides, con plage suprahilar no amiloide. 
Basidios (Fig. 5B) 33,0-55,0 × 8,5-11,5 $\mu \mathrm{m}$, claviformes a subclaviformes, 4-esporados. Pleurocistidios (Fig. 5C) 50-80 × 7-10 $\mu \mathrm{m}$, cilíndricos a fusiformes, anchos en la porción baja, abundantes. Queilocistidios (Fig. 5D) 25,0-45,0 x 5,0- 7,7 $\mu \mathrm{m}$, similares a pleurocistidios, frecuentemente con vértice submucronado, abundantes. Pileipellis (Fig. 5E) una trichodermis, no gelificada, con hifas emergentes de 10-40 x 2-5 $\mu \mathrm{m}$; subpellis con hifas isodiamétricas, hasta
$12 \mu \mathrm{m}$ de diám. Estipitipellis hifas entrelazadas irregularmente de donde emergen elementos terminales similares a los de la pileipellis. Fíbulas presentes.

Hábito. Solitario o gregario, creciendo sobre suelo, entre hojarasca y pinocha de Pinus radiata, a veces sobre su madera muerta con alto grado de descomposición y humedad. Coleccionado generalmente en épocas de abundante lluvia y humedad.

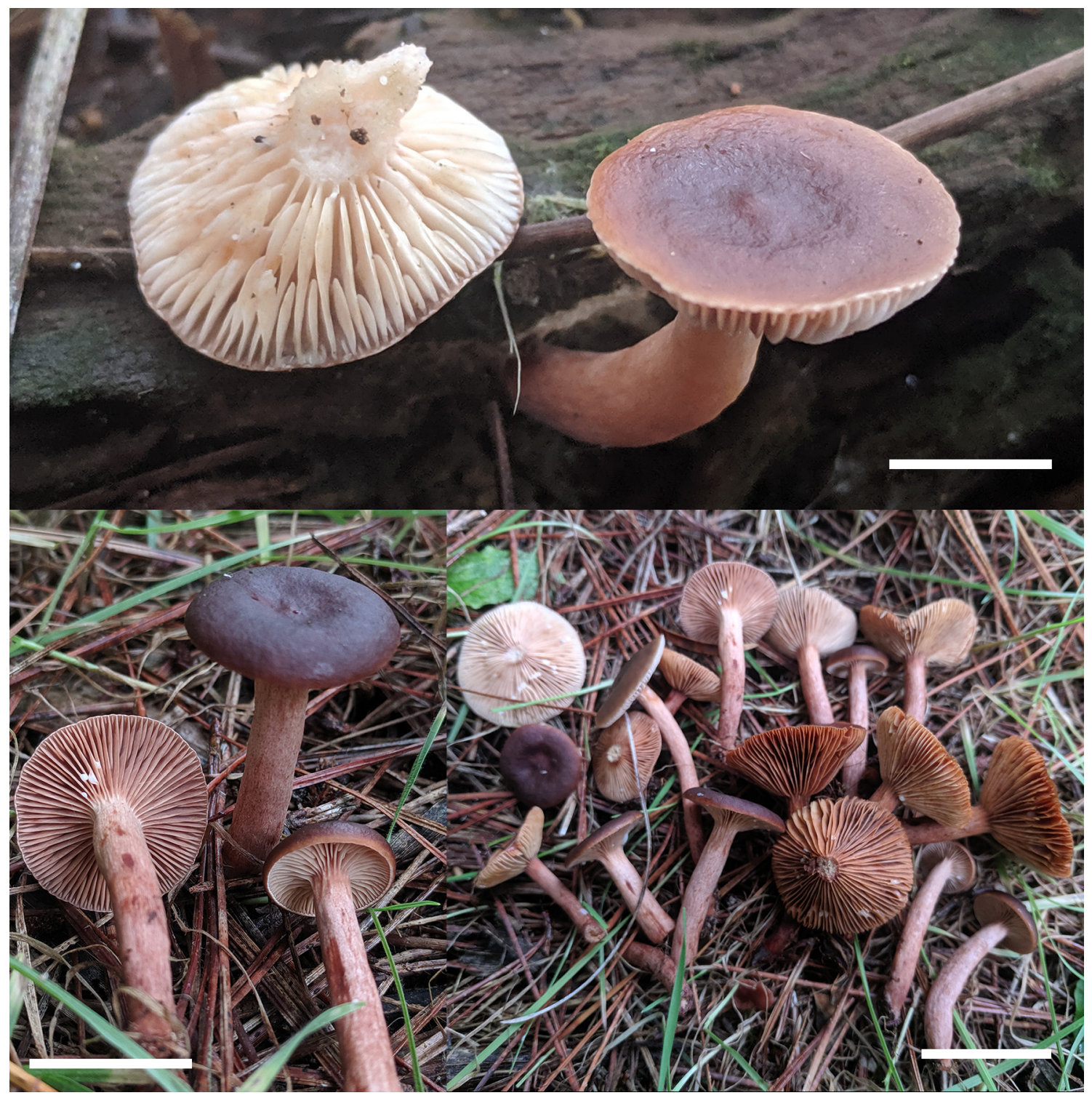

Fig. 4. Lactarius hepaticus. Aspecto general y presencia de látex sobre las laminillas. Escala: 15 mm. Fotos Agustín P. Martínez. Figura en color en la versión en línea http://www.ojs.darwin.edu.ar/index.php/darwiniana/article/view/888/1191 
Distribución y hábitat. Bosques de pinos, micorriza con pino insigne (Pinus radiata), pinos resineros (Pinus pinaster) y piñoneros (Pinus pinnea), generalmente en España y Francia (Pierotti, 2007). También se encuentra distribuida en gran parte del hemisferio norte de América (Hesler \& Smith, 1979). Por tratarse de un género micorrícico asociado a especies del género Pinus, su hallazgo en la Argentina puede deberse a una introducción junto a Pinus spp.

Observaciones. L. hepaticus se caracteriza por tener un píleo castaño oscuro, color similar al hígado, ser higrófano, una superficie suave con una textura un poco más rugosa hacia el centro. Genera un látex blanco que vira lentamente al amarillo y está asociado a Pinus spp.

Existen dentro del género especies similares, aunque con caracteres que las diferencian. L. decipiens Quél., distinguible por su olor característico a geranios, presenta un sabor no picante y tonalidades rosadas en el píleo (Pierotti, 2007; Leonard, 2008). L. obscuratus (Lasch) Fr., generalmente asociado a Alnus spp., y L. lacunarum Romagn. ex Hora presentan un píleo estriado de tonalidades rojizas más oscuras que $L$. hepaticus, (Courtecuisse \& Duhem, 1995; Watling \& Turnbull, 1998; Pierotti, 2007). L. atrobadius Hesler \& A.H. Sm., L. subdulcis (Pers.) Gray, L. omphaliiformis Romagn. y L. rubidus (Hesler \& A.H. Sm.) Methven, con un olor dulce, similar al jarabe de arce (Kränzlin, 2005), se diferencian por tener un látex blanco inmutable (Romagnesi, 1938; Hesler \& Smith, 1979; Methven, 1997; Phillips, 2006). L. camphoratus (Bull.) Fr., distinguible por la ausencia de un plage suprahilar amiloide, presenta olor fuerte a achicoria, y fructifica mayormente en zonas montañosas (Marchand, 1980; Leonard, 2008).

Según Hansen \& Knudsen (1993), HeilmannClausen et al. (1998) y Basso (1999), las especies más similares son L. basidiosanguineus Kühner \& Romagn. y L. sphagneti (Fr.) Neuhoff, aunque se diferencian por encontrarse en ambientes más hidrofílicos, poseer cutícula brillosa, tonalidades más rojizas, esporas con ornamentación más evidente y un látex blanco inmutable. L. hepaticus es difícil de confundir dentro de la sección Pseudoaurantiaci debido a que las otras especies se encuentran relacionadas a árboles latifoliados (Pierotti, 2007).

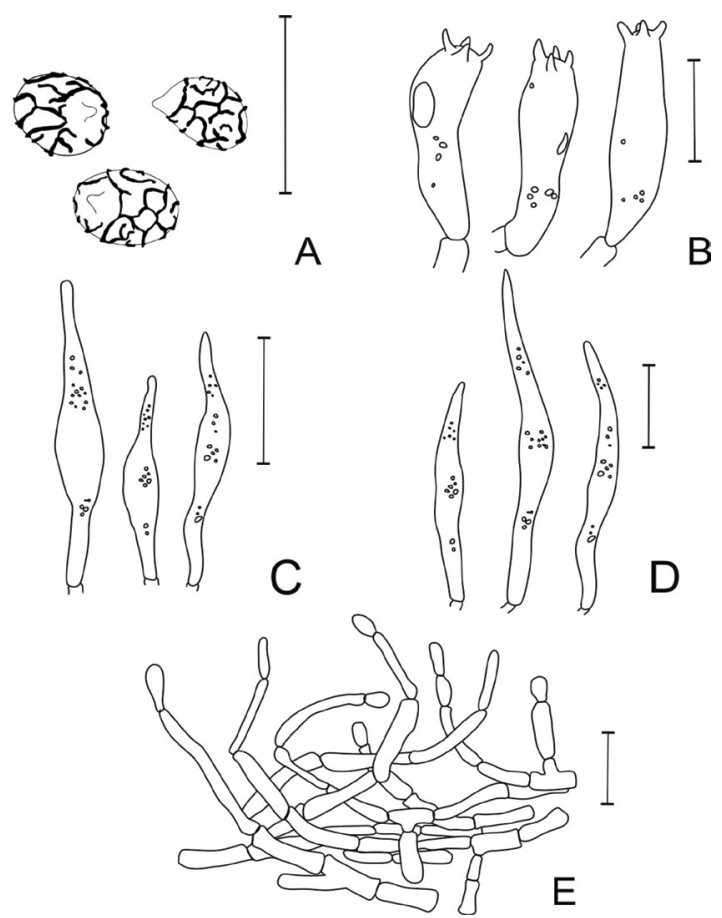

Fig. 5. Lactarius hepaticus. A, esporas. B, basidios. C, pleurocistidios. D, queilocistidios. E, pileipellis. Escala: $20 \mathrm{~mm}$. Ilustraciones Juan M. Suarez.

\section{Material estudiado}

ARGENTINA, Buenos Aires, Partido de la Costa, Aguas Verdes, $36^{\circ} 38^{\prime} 43^{\prime \prime} \mathrm{S} 56^{\circ} 41^{\prime} 16^{\prime \prime}$ O, 15-jun.-19, Leg. J. M. Suarez \& A. P. Martínez, BAFC 53416; Costa del Este, $36^{\circ} 37^{\prime} 5^{\prime \prime} \mathrm{S} 56^{\circ}$ 41' 9" O, 15-jun.-19, Leg. J. M. Suarez \& A. P. Martínez, BAFC 53415.

Mycena margarita (Murrill) Murrill, Mycologia 8: 220. 1916. Prunulus margarita Murrill, N. Amer. Flora 9: 340. 1916. Prunulus subepipterygius Murrill, Bull. Torrey Bot. Club 67: 233. 1940. Mycena subepipterygia (Murrill) Murrill, Bull. Torrey Bot. Club 67:235. 1940. Mycena chlorinosma Singer, Revue Mycol. 2:232. 1937. TIPO: JAMAICA, $1500 \mathrm{~m}$ de elevación, "on decayed wood under tree ferns at Morce's Gap"; 29, 30 Dic., 2 Jan 1908-1909, W.A. Murrill and E. Murrill 731 (Herbario N. Y. Botanical Garden). Fig. 6-7. 
Píleo (Fig. 6) 15-30 mm de diám., al principio cónico-campanulado, luego plano-convexo con un umbón ligeramente deprimido en el centro, superficie blanquecina-grisácea (5B1) con tonalidades castaño grisáceas (6D6) hacia el centro y estrías radiales del mismo color, higrófano, subvícido a víscido. Los primeros estadios del píleo presentan tonalidades castaño oscuras (7E6). Carne delgada, menor a $2 \mathrm{~mm}$. Laminillas adnexas a adnatas, subdistantes, ligeramente anchas, con lamélulas de dos tamaños, blancas (5A1). Estípite 10-20 x 1-3 mm, central, cilíndrico, blanquecino grisáceo (5B2), aclarándose hacia el ápice (5B1), disco basal de color ocre-grisáceo (6D4). Esporada blanca-blanquecina. Olor levemente cloroso o no distintivo, la intensidad es variable entre las distintas colecciones, generalmente muy leve. Sabor no distintivo. Comestibilidad desconocida, sin valor culinario, carne escasa. Bioluminiscencia no observada. Esporas (Fig. 7A) (5,5) 6,4-8,8 x 3,4$5,0 \mu \mathrm{m} ; \mathrm{Me}=7,5 \times 4,2 \mu \mathrm{m} ; \mathrm{N}=52 ; \mathrm{Q}=1,6-2$; $\mathrm{Qe}=1,7$; elipsoidales, lisas, hialinas, pared delgada, débilmente amiloides. Basidios (Fig. 7B) 14,5-19,0 $\mathrm{x}$ 7,5-9,5 $\mu \mathrm{m}$, claviformes, 4-esporados, fíbulados.

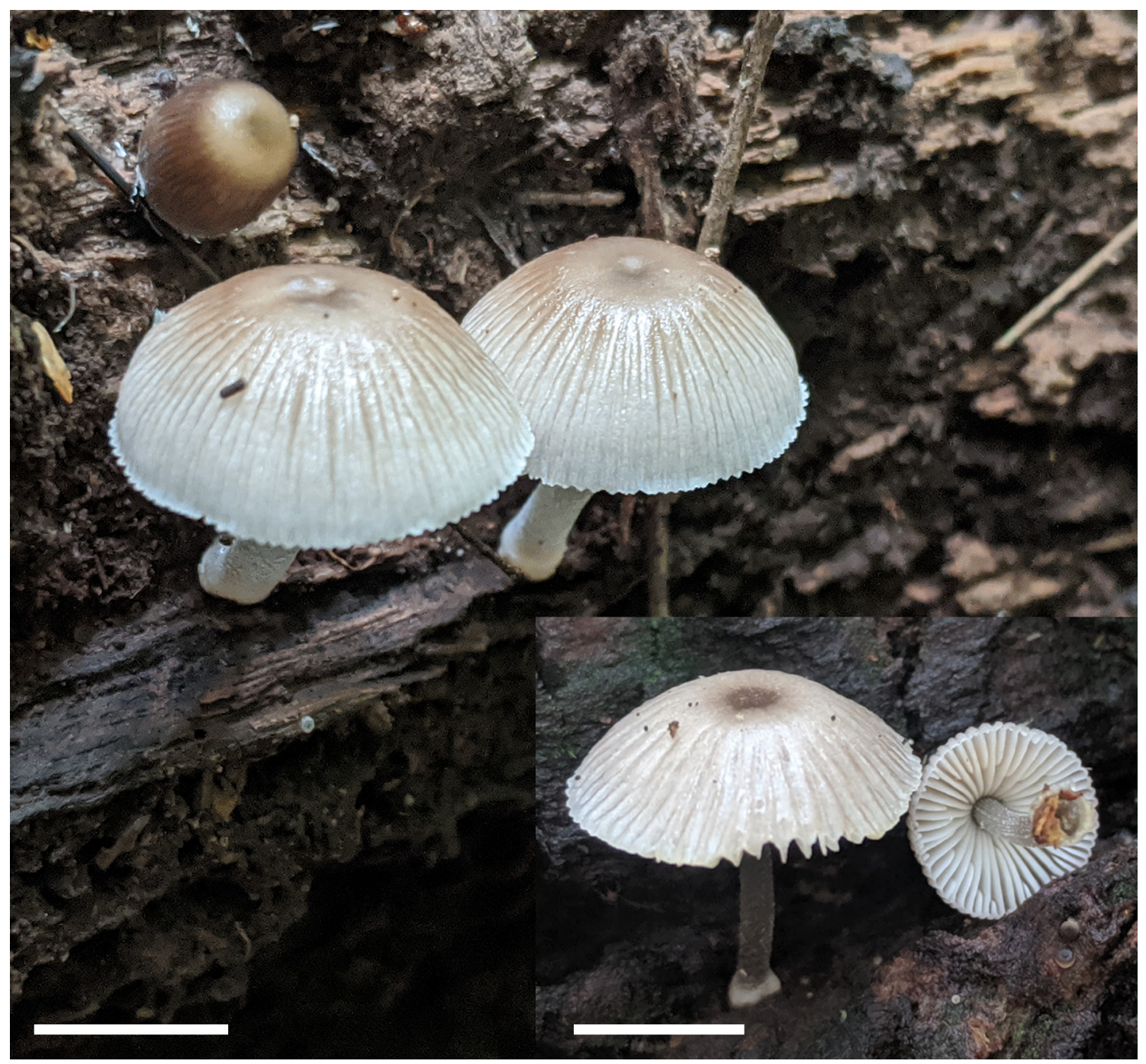

Fig. 6. Mycena margarita. Aspecto general. Escala: $10 \mathrm{~mm}$. Fotos Agustín P. Martínez. Figura en color en la versión en línea http://www.ojs.darwin.edu.ar/index.php/darwiniana/article/view/888/1191 
Pleurocistidios ausentes. Queilocistidios (Fig. 7C) 30-60 × 9-18 $\mu \mathrm{m}$, fusoides-ventricosos a claviformerostrados, lisos, ápice delgado, cónico simple o bífido-ahorquillado, hialinos, inamiloides, de pared delgada. Trama himenoforal regular, formada por hifas cortas, no gelatinosas, dextrinoides y de pared delgada. Pileipellis (Fig. 7E) un ixocutis (180-250 $\mu \mathrm{m}$ de grosor), compuesto por hifas estrechas, hialinas, de pared delgada, inamiloides, 1,5-3,0 $\mu \mathrm{m}$ de diám., elementos terminales (Fig. 7F) 20-43 x 7-15 $\mu \mathrm{m}$, claviformes a sub-cilíndricos, espinulados, hialinos, inamiloides, pared delgada. Hifas del contexto del píleo entrelazadas, cilíndricas, pared delgada, no gelatinosas, hialinas, dextrinoides, 3-16 $\mu \mathrm{m}$ de diám. Estipitipellis un cutis formado por hifas corticales, 3-8 $\mu \mathrm{m}$ de diám., no gelatinosas, paralelas, lisas, cilíndricas, hialinas, inamiloides, dextrinoides. Caulocistidios (Fig. 7D) 50-80 x 7-14 $\mu \mathrm{m}$, fusoides a lanceolados o ventricosos, hialinos, pared delgada, inamiloides, no gelatinosos, solitarios o en racimos. Fíbulas presentes en todos los tejidos.

Hábito. Solitario o gregario, creciendo sobre madera muerta con alto porcentaje de humedad. Coleccionado generalmente en épocas de abundante lluvia y humedad.

Distribución y hábitat. Su distribución abarca el sur de Estados Unidos (Florida) hasta Brasil, aunque generalmente se encuentra en Belice, República Dominicana, Dominica, Honduras, Jamaica, Puerto Rico y Venezuela (Murrill, 1916a; 1916b; Smith, 1947; Dennis, 1951; Pegler, 1983; Desjardin et al., 2010). El hallazgo de esta especie en Argentina amplía su distribución en el continente americano.

Observaciones. M. margarita se caracteriza por poseer una zona bulbosa en la base del estípite, pileipellis en un ixocutis de 150-200 $\mu \mathrm{m}$ de espesor y bioluminiscencia leve o nula según la población.

Desjardin et al. (2010) realizaron una comparación de las características microscópicas de varias colecciones de $M$. margarita, observando una gran variabilidad en la reacción amiloide y tamaño de las esporas, olor e intensidad y tonalidad de bioluminiscencia. Alves \& Nascimento (2014) describió la primera cita para Brasil, indicando las mismas características microscópicas, coincidentes con nuestras observaciones.
Las esporas de las colecciones estudias en este trabajo mostraron una reacción amiloide muy leve y un largo mayor que las observadas por Desjardin et al. (2010). Sin embargo, en las descripciones de Smith (1947) y Dennis (1951) se citan medidas similares a las aquí reportadas. La bioluminiscencia no se pudo observar y ninguna muestra presentó algún olor característico.

Mycena margarita es muy similar macromorfológicamente a $M$. chlorophos, pero se diferencian por una bioluminiscencia más leve, la pileipellis gelatinosa más gruesa $(150-200 \mu \mathrm{m}$ vs $80 \mu \mathrm{m}$ en $M$. chlorohos), queilocistidios de menor longitud (30-60 x 9-18 $\mu \mathrm{m}$ vs. $100 \times 11$ $22 \mu \mathrm{m}$ en $M$. chlorohos), y esporas generalmente más pequeñas $(6,9$ x 4,4 $\mu \mathrm{m}$ vs 7,8 x 5,4 $\mu \mathrm{m}$ en $M$. chlorophos) (Desjardin et al., 2010).

Por otro lado, Desjardin et al. (2010), basados en Singer (1937), Murrill (1940) y Maas Geesteranus (1989), aceptan Prunulus subepipterygius y Mycena subepipterygia como sinónimo de M. margarita, y M. chlorinosma como posible sinónimo.
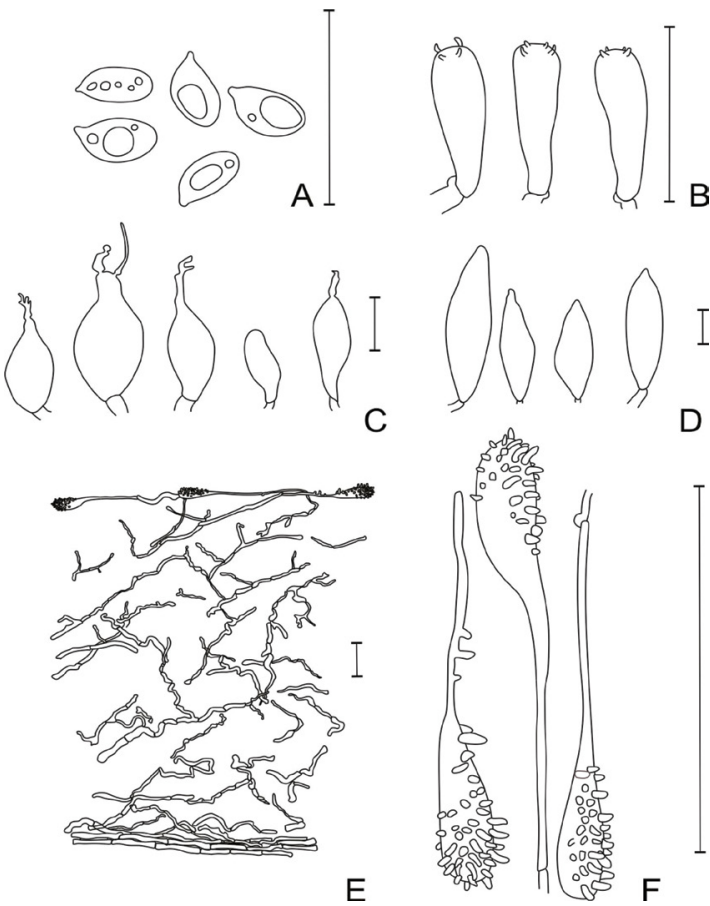

Fig. 7. Mycena margarita. A, esporas. B, basidios. C, queilocistidios. D, caulocistidios. E, pileipellis. F, elementos marginales del píleo. Escala: $20 \mathrm{~mm}$. Ilustraciones Juan M. Suarez. 


\section{Material estudiado}

ARGENTINA, Buenos Aires, Lomas de Zamora, Reserva Santa Catalina, $34^{\circ} 47^{\prime} 8^{\prime \prime} \mathrm{S} 58^{\circ}$ 26' 34" O, 11-sept.-18, Leg. J. M. Suarez, BAFC 53408; Moreno, Reserva Los Robles, 34 39' 47', S 58 51'30" O, 11-nov.-19, Leg. A. P. Martínez, BAFC 53405; Reserva Los Robles, 34 39' 42" S 58 51'30" O, 23-oct.-19, Leg. A. P. Martínez, BAFC 53406; $I b$. 34 39' 42" S 58 51' 54" O, 4-nov.-19, Leg. A. P. Martínez, BAFC 53407; Ib. 34 39' 36" S 58 51'36” O, 20-abril-2020, Leg. A. P. Martínez, BAFC 53408; Partido de la Costa, San Bernardo, $36^{\circ} 22^{\prime} 36^{\prime \prime} \mathrm{S} 56^{\circ} 42^{\prime} 49^{\prime \prime}$ O, 14-jun.-19, Leg. J. M. Suarez \& A. P. Martínez, BAFC 53409.

\section{AGRADECIMIENTOS}

A la Universidad de Buenos Aires y el CONICET por el financiamiento y disponibilidad de equipamiento. A Diego Higer por su ayuda bibliográfica, al personal del Parque Municipal Los Robles, en especial a Ignacio Healion, por el apoyo en la investigación y a Susana Pereira por su amable disponibilidad en incorporar las colecciones al BAFC en el momento de la pandemia COVID-19.

\section{BIBLIOGRAFÍA}

Albertó, E. 1998. A new species of Agaricus from Argentina. Mycotaxon 66: 205-213.

Alves, M. H. \& C. C. Nascimento. 2014. Mycena margarita (Murrill) Murrill, 1916 (Basidiomycota: Agaricales: Mycenaceae): A bioluminescent agaric first recorded in Brazil. Check List 10(1): 239-243.

Arnold, N. 1993. Morphologisch-anatomische und chemische Untersuchungen an der Untergattung Telamonia (Cortinarius, Agaricales). Libri botanici vol 7, p. 44. Eching, Deutschland.

Basso, M. T. 1999. Lactarius, sottogenere Plinthogalus nell'Herbarium Giacomo Bresadola. Bollettino del Gruppo micologico G. Bresadola, nuova serie 40 (2-3): 47-58.

Courtecuisse, R. \& B. Duhem. 1995. Mushroom and Toadstools of Britain and Europe. HarperCollins, London.

Dennis, R. W. G. 1951. Some Agaricaceae of Trinidad and Venezuela. Leucosporae I. Transactions of the British Mycological Society 34: 411-482.
Desjardin, D. E.; B. A. Perry, D. J. Lodge, C. V. Stevani \& E. Nagasawa. 2010. Luminescent Mycena: new and noteworthy species. Mycologia 102: 459-477. DOI: https:// doi.org/10.3852/09-197

Hansen, L. \& H. Knudsen. 1993. Nordic Macromycetes Vol. 2. Polyporales, Boletales, Agaricales, Russulales. Nordvamp, Copenaghen. 474 pp.

Heilmann-Clausen, J.; A. Verbeken \& J. Vesterholt. 1998. The genus Lactarius. Fungi o Northen Europe Vol. 2. Low Budget Publishing, Sabon. 287 pp.

Hesler, L. R. \& A. H. Smith. 1979. North American Species of Lactarius. University of Michigan Press, Ann Arbor.

Horak, E. 1980. Fungi Basidiomycetes. Agaricales y Gasteromycetes Secotioides. Flora Criptogámica de Tierra del Fuego 11(6): 1-528.

Kornerup, A., \& J. H. Wanscher. 1987. Methuen handbook of colour. ISBN 0413334007.

Kränzlin, F. 2005. Fungi of Switzerland. Volume 6. Verlag Mykologie, Lucerne.

Largent, D. L. 1986. How to Identify Mushrooms to Genus I Macroscopic Features. Mad River. Eureka, California.

Lechner, B. E.; E. Albertó, R. Petersen. 2003. Pleurotus araucariicola is Pleurotus pulmonarius. Mycotaxon: 86: 425-431.

Lechner, B. E.; J. E. Wright \& E. Albertó E. 2004. The genus Pleurotus in Argentina. Mycologia: 96 (4): 844-857.

Lechner, B. E.; J. E. Wright \& E. Albertó. 2005. The genus Pleurotus in Argentina: mating tests. Sydowia 57 (2): 233245.

Lechner, B. E.; J. E. Wright \& O. Popoff. 2006. New taxa and new records for Argentina of fungi from Iguazú National Park, Misiones. Fungal diversity 21: 131-139.

Lechner, B. E. \& E. Albertó. 2008. Especies tóxicas de Agaricales halladas en la Argentina: nueva cita de Amanita pantherina y reevaluación de la comestibilidad de Tricholoma equestre. Boletín de la Sociedad Argentina de Botánica. 43 (3-4): 227 - 235.

Leonard, P. 2008. Synoptic keys to British species of Lactarius. British Mycological Society Keys. United Kingdom. p. 27.

Maas Geesteranus, R. A. 1989. Conspectus of the Mycenas of the northern hemisphere 12. Proceedings of the Koninklijke Nederlandse Akademie van Wetenschppen 92: 331-365.

Marchand, A. 1980. Champignons du Nord et du Midi. Tome 6. Lactaires et Pholiotes. Hachette, Perpignan.

Methven, A. S. 1997. The Agaricales (Gilled Fungi) of California. 10. Russulaceae II. Lactarius. Mad River Press, Eureka.

Moënne-Loccoz, P. \& P. Reumaux, 1990. Atlas des Cortinaires, pars II. Dauphiné-Savoie, France. Bulletin trimestriel Fédération Mycologique Dauphiné-Savoie 28 (111): 23-26. 
Moser, M. \& E. Horak. 1975. Cortinarius Fr. Und nahe verwandte gattungen in Südamerika. Beihefte zur Nova Hedwigia 52: 1-628.

Moser, M. 2001. Some aspects of Cortinarius associated with Alnus. Journal des JEC, Journées Européenes du Cortinaire 4(3): 47-101.

Mueller, G. M. \& Q. X. Wu. 1997. Mycological contributions of Rolf Singer: field itinerary, index to new taxa, and list of publications. Fieldiana, Botany, 38: 124.

Murrill, W. A. 1916a. Prunulus. North American Flora 9 (5): 319-344.

Murrill, W. A. 1916b. Pleurotus, Omphalia, Mycena and Collybia published in North American Flora. Mycologia 8: 218-221.

Murrill, W. A. 1940. Additions to Florida fungi IV. Bulletin of the Torrey Botanical Club 67: 227-235.

Niskanen, T.; I. Kytövuori \& K. Liimatainen. 2009. Cortinarius sect. Brunnei (Basidiomycota, Agaricales) in North Europe. Mycological Research 113: 182-206.

Niveiro, N. \& E. O. Albertó. 2012. Checklist of the Argentine Agaricales 4. Tricholomatacea \& Polyporaceae. Mycotaxon 121: 499. DOI: https://doi.org/10.5248/121.499

Niveiro, N. \& E. O. Albertó. 2013; Checklist of the Argentine Agaricales 6. Paxillaceae, Gomphidiaceae, Boletaceae, and Russulaceae. Mycotaxon 123: 491.

Niveiro, N. \& E. O. Albertó. 2014; Checklist of the Argentine Agaricales 7. Cortinariaceae and Entolomataceae; CheckList 10: $72-96$.

Pegler, D. N. 1983. The Agaric flora of Lesser Antilles. Kew Bulletin Additional Series 9: 1-668.

Phillips, R. 2006. Mushrooms. Macmillan, London.

Pierotti, A. 2007. Contributo alla conoscenza del genere Lactarius. 2. Lactarius hepaticus. Bollettino del Gruppo Micologico G. Bresadola di Trento, nuova serie: 48: 47-56.

Romagnesi, H. 1938. A la recherche de Lactarius subdulcis. Bulletin Trimestriel de la Société Mycologique de France 54: 204235.
Romano, G. M. \& B. E. Lechner. 2013. The Cortinariaceae of Argentina's Nothofagus forests. Mycotaxon 126: 247.

Salgado Salomón, M. E.; P. Dresch, E. Horak, F. Galleguillos, C. Barroetaveña \& U. Peintner. 2018. The enigmatic Cortinarius magellanicus complex occurring in Nothofagaceae forests of the Southern Hemisphere. Fungal Biology 122(11): 1077-1097. DOI: https://doi. org/10.1016/J.FUNBIO.2018.08.009

Singer R. 1937. Notes sur quelques Basidiomycetes. Revue Mycologique 2: 226-242.

Singer, R. 1954. Agaricales von Nahuel Huapi. Sydowia 8: 100157.

Singer, R. 1969. Mycoflora australis. Beihefte Nova Hedwigia 29: 1-405.

Singer, R. \& A. P. L. Digilio. 1952. Pródromo de la flora agaricina Argentina. Lilloa 25: 6-461.

Smith, A. H. 1947. North American species of Mycena. North American species of Mycena. Ann Arbor: Univ Michigan Press. 521 p.

Spegazzini, C. 1887. Fungi patagonici. Boletín de la Academia Nacional de Ciencias de la República Argentina 11(1): 5-64.

Spegazzini, C. 1888. Fungi fuegiani. Boletín de la Academia Nacional de Ciencias de la República Argentina 11(2): 135308.

Spegazzini, C. 1899. Fungi argentini novi v. critici. Anales del Museo Nacional de Buenos Aires 6: 6-365.

Suárez-Santiago, V. N.; A. Ortega, U. Peintner \& I. LópezFlores. 2009. Study on Cortinarius subgenus Telamonia section Hydrocybe in Europe, with especial emphasis on Mediterranean taxa. Mycological Research. 113: 10701090. DOI: https://doi.org/10.1016/j.mycres.2009.07.006

Watling, R. \& E. Turnbull. 1998. British Fungus Flora Volume 8. Royal Botanic Gardens, Edinburgh.

Wright, J. E. \& E. Albertó. 2002. Guía de hongos de la región pampeana. I. Hongos con laminillas. L.O.L.A., Buenos Aires. 